\title{
Konzeption und Evaluierung eines Laboraufbaus zur photoakustischen Detektion von CH4 mit modulierter Breitband MEMS-IR-Strahlungsquelle
}

\author{
B. Vatter ${ }^{1,2}$, A. Magi ${ }^{1}$, F. Liebold ${ }^{1,3}$ \\ ${ }^{1}$ Micro-Hybrid Electronic GmbH, Forschung und Entwicklung, 07629 Hermsdorf \\ ${ }^{2}$ Westsächsische Hochschule Zwickau, Fakultät für Physik und Informatik, 08056 Zwickau \\ ${ }^{3}$ Duale Hochschule Gera-Eisenach, Fachbereich Technik, 07546 Gera \\ benedikt.vatter.f4u@fh-zwickau.de, a.magi@micro-hybrid.de,falk.liebold@dhge.de
}

\section{Zusammenfassung}

In dieser Arbeit wird eine photoakustische Messzelle zur Bestimmung von Methan vorgestellt. Die Grundlage stellt eine kommerzielle NDIR-Gasmesszelle dar, die um ein MEMS-Mikrofon erweitert wurde. Zur Erzeugung des photoakustischen Signals wurde ein MEMS-Breitband-IR-Strahler bei einer Frequenz von $9 \mathrm{~Hz}$ elektrisch moduliert. Mittels Lock-In-Verfahren wurde das Signal in Abhängigkeit der Konzentration von Methan detektiert. Das zu erwartende Signal konnte über eine rechnerische Modellgleichung abgeschätzt und so mit den experimentellen Werten verglichen werden. Es wird gezeigt, dass diese Abschätzung mit den experimentellen Daten gut übereinstimmt und somit prinzipiell für die Realisierung von photoakustischen Messzellen verwendet werden kann. Der Sensor wurde mit zwei Strahlertypen erprobt. Es wurde ein Detektionslimit von 108 ppmV bei einer Auflösung von 43 ppmV ermittelt.

Keywords: Gassensorik, photoakustische Spektroskopie, Methan, Absorptionsspektroskopie, MEMSMembranstrahler, NDIR, IR-Strahler, Hot-plate Emitter

\section{Einleitung}

Die Bestimmung der Konzentration IR-aktiver Gase wie z.B. $\mathrm{CO}_{2}$ und $\mathrm{CH}_{4}$ spielt in der Umwelt- und Prozessüberwachung eine tragende Rolle. Als Messprinzip bietet sich neben der weit verbreiteten NDIRSpektroskopie die Photoakustik an. Es wurde bereits mehrfach gezeigt [1][2][3], dass die Photoakustik für die Bestimmung geringster Konzentrationen bis in den unteren pptVBereich geeignet ist. Jedoch sind diese Methoden oft sehr komplex und nur für den Laboraufbau geeignet. Für die einfache Integration in bereits bestehende Gassensoren und zur Minimierung mechanischer Bauteile, wurde in dieser Arbeit ein Messzellenkonzept aus elektrisch moduliertem MEMSBreitbandstrahler und einem MEMS-Mikrofon untersucht. Das zu erwartende photoakustische Signal für Methan wurde im Vorfeld durch eine geeignete Modellgleichung abgeschätzt.

\section{Grundlagen}

Das photoakustische Prinzip wurde erstmals 1880 von A. G. Bell erwähnt. Es beschreibt die Erzeugung einer Schallwelle durch einen modulierten Energieeintrag, in Form von Strahlung, in ein System [4]. Die periodisch eingebrachte Energie $Q$ aus einer
Strahlungsquelle führt nach GI. (1) zu einer Änderung der Temperatur $\Delta T$ und nach der idealen Gasgleichung GI. (2) auch zu einer Druckänderung $\Delta p$ [5]. Aus der optischen Leistung der Strahlungsquelle ergibt sich die in das System eingebrachte Wärmeenergie pro Zeitintervall.

$$
\begin{gathered}
Q=C_{V} \Delta T \\
\Delta p=\frac{n R \Delta T}{V}
\end{gathered}
$$

Diese Druckänderung ist abhängig von den Eigenschaften der Strahlungsquelle, dem Absorptionsverhalten des zu untersuchenden Materials, der Geometrie der Messzelle und der Konzentration des Stoffes. Damit lässt sich für kleine Konzentrationen c die Druckänderung nach $\mathrm{Gl}$. (3) berechnen.

$$
\Delta p=\frac{R P_{o p t} \alpha l c d c m \delta T_{o p t}}{c_{V} V_{\text {Zelle }} f}
$$

Die Druckänderung ergibt sich aus der idealen Gaskonstante R, der optischen Leistung der 
Strahlungsquelle $\mathrm{P}_{\text {opt, }}$ dem Absorptionskoeffizienten $\alpha$, der Länge I und dem Volumen Vzelle der Messzelle. Weiterhin geht der duty cycle dc (Verhältnis zwischen An- und Auszeit der Strahlungsquelle), der IntensitätsModulationsgrad $\mathrm{m}$ des Strahlers (Verhältnis der Intensität im An-Zustand zur max. möglichen Intensität im unmodulierten Betrieb), sowie ein Korrekturfaktor $\delta$, der die Überlagerung von Emissionspektrum der Strahlungsquelle und Absorptionsspektrum der Probe berücksichtigt mit ein. Auch der Transmissionskoeffizient $T_{\text {opt }}$ der optischen Komponenten im Strahlengang, die spezifischen Wärmekapazität $C_{v}$ sowie die Frequenz $f$ der modulierten Strahlungsquelle werden in der verwendeten Modellgleichung Gl. (3) berücksichtigt. Die daraus resultierenden Ergebnisse wurden zur Abschätzung im Vorfeld der eigentlichen Messung verwendet. Vorgänge bei der Absorption und Verlustmechanismen, wie sie in [6][7] diskutiert werden, sollen hier nicht weiter betrachtet werden.

\section{Vorstellung der Komponenten}

Im Vorfeld wurden die Eigenschaften der Strahlungsquelle, der Messzelle und der Probe untersucht. Als Strahlungsquelle kommt ein MEMS-Membranstrahler JSIR-350 der Firma Micro-Hybrid Electronic GmbH (MHE) zum Einsatz. Das Emissionsspektrum bei 0,8 W elektrischer Leistung ist in Abb. 1 dargestellt.

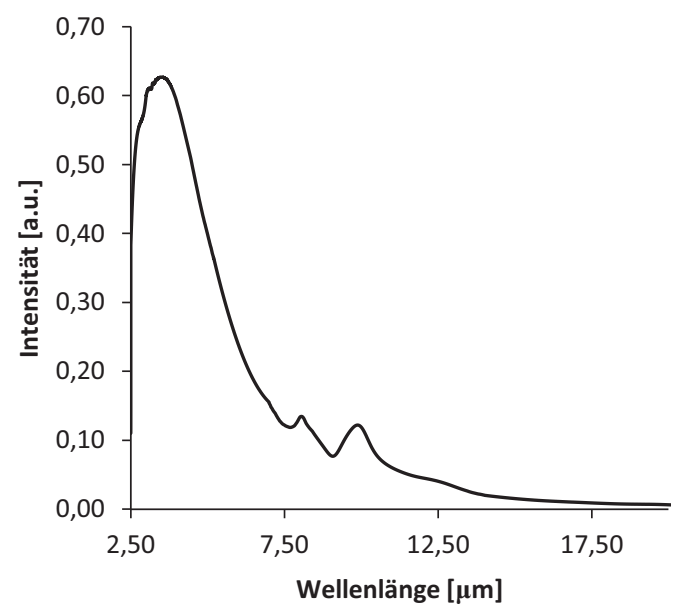

Abb. 1: Normiertes Emissionsspektrum des MEMS-Breitbandstrahlers JSIR-350 zwischen 2,5 und $20 \mu \mathrm{m}$.

Der Strahler wird sowohl offen als auch mit Krypton $(\mathrm{Kr})$ verschlossen eingesetzt. Der mit $\mathrm{Kr}$ verschlossene Strahler kann, aufgrund der schlechteren Wärmeleitung gegenüber Luft, bei gleicher elektrischer Leistung höhere Temperaturen und damit auch höhere optische Leistungen erzielen. $\mathrm{Da}$ es sich um einen thermischen Dünnschicht- bzw. MembranStrahler handelt, kann dieser mit einer Frequenz im Hz-Bereich betrieben werden. Mit Modulationsfrequenzen größer $20 \mathrm{~Hz}$ nimmt der Modulationsgrad $\mathrm{m}$ merklich ab. Zur Detektion wurde daher ein MEMS-Mikrofon ICS-40300 der Firma Invensense verwendet, welches bis $6 \mathrm{~Hz}$ sensitiv ist.

Als Messkammer dient eine NDIRGasmesszelle der Firma MHE (Abb. 2). Der Absorptionspfad ist $10 \mathrm{~cm}$ lang und besitzt einen Radius von $4 \mathrm{~mm}$. Damit ergibt sich ein Zellvolumen von rund $5 \mathrm{ml}$. Zu- und Abfluss der Zelle können über Magnetventile verschlossen werden, um Störgeräusche aus der Leitung zu minimieren.

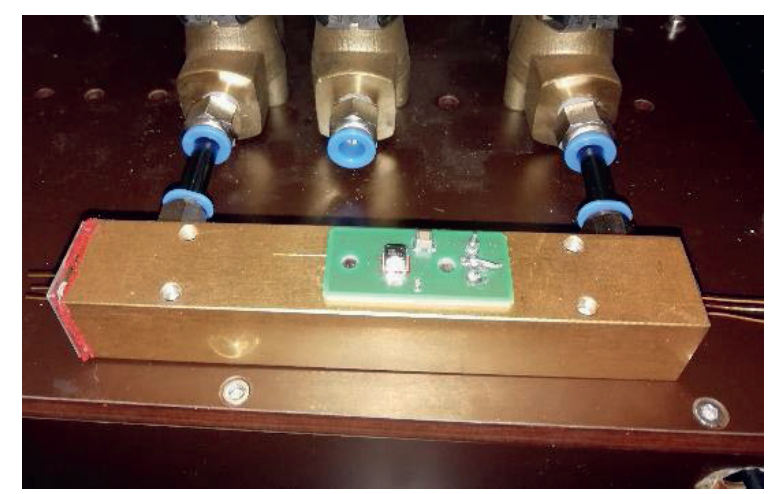

Abb. 2: NDIR-Gasmesszelle mit zusätzlichem MEMS-Mikrofon. Zuund Abfluss über Magnetventil regelbar.

Die Mikrofonspannung wurde mittels LocklnVerstärker (LIA, 5210 Dual Phase Analog Lockin Amplifier Fa. Signal Recovery) um Faktor $10^{4}$ verstärkt und mit einem erstellten LabVIEWProgramm ausgelesen. Das Ansteuersignal des Strahlers diente als Referenzsignal. Die Integrationszeit des LIA betrug $3 \mathrm{~s}$.

Zur Bestimmung des Optimums aus Modulationsfrequenz der Strahlungsquelle und Empfindlichkeit des Mikrofons, wurde die Messzelle zunächst mit $\mathrm{CO}_{2}$ befüllt und der Frequenzgang bis $15 \mathrm{~Hz}$ aufgenommen. Wie in Abb. 3 zu erkennen ist, tritt ein Maximum des Sensorsignals bei $9 \mathrm{~Hz}$ auf. Die indirekte Proportionalität der Anregungsfrequenz führt zu einer Minimierung des Schallwechseldrucks für Frequenzen größer $9 \mathrm{~Hz}$ (siehe GI. 3). Bei höheren Frequenzen führt auch der sinkende Modulationsgrad $\mathrm{m}$ des Strahlers selbst zu einer Abnahme des Ausgangssignals.

\section{Messung}

Als Probe wird Methan verwendet. $\mathrm{CH}_{4}$ weist eine gute Überlagerung der Absorptionsbanden 
mit dem Maximum des Emissionsspektrums der Strahler auf. Da kein optischer Filter verwendet wurde, tragen alle Absorptionsbanden, die mit der Strahlungsquelle angeregt werden können zur Schallgenerierung bei. Für gasspezifische Anwendungen können zur Minimierung von Quereinflüssen auf spezielle Absorptionsbanden angepasste Filtergläser verwendet werden.

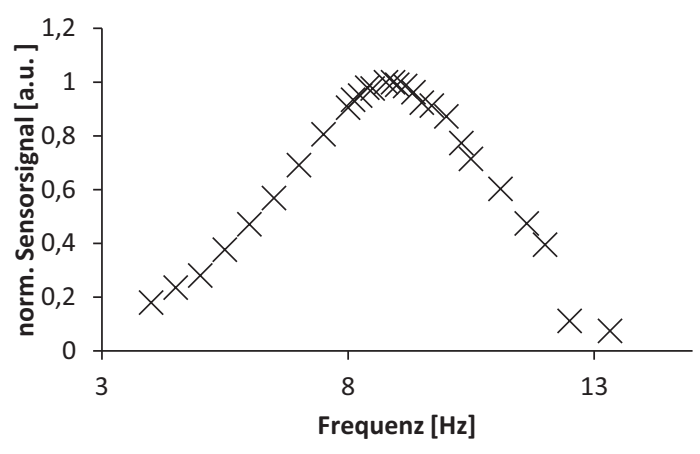

Abb. 3: Frequenzgang des photoakustischen Sensors. Maximales Signal bei $9 \mathrm{~Hz}$.

Zur Charakterisierung des Messsystems wurde eine Messreihe von 0-1073 ppmV $\mathrm{CH}_{4}$ in $\mathrm{N}_{2}$ verdünnt aufgenommen. Dabei wurden die Messungen mit einem offenen und mit einem $\mathrm{Kr}$ gefüllten Strahler untersucht. Die Zelle wurde vor jeder Messung mit $\mathrm{N}_{2}$ gespült und danach 4 min mit dem $\mathrm{CH}_{4}-\mathrm{N}_{2}-$ Gemisch durchspült. Nachdem die Messzelle vollständig mit dem Messgas geflutet war, wurden die Magnetventile verschlossen und das photoakustische Signal detektiert. Das zeitliche Verhalten des Signals zeigt Abb. 4.

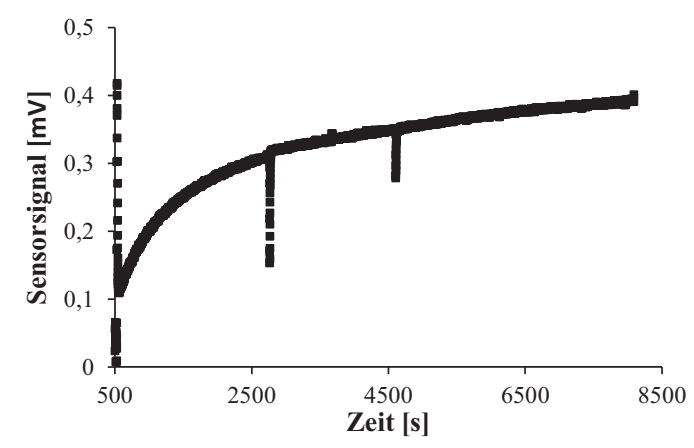

Abb. 4: Zeitlicher Verlauf des photoakustischen Signals für 4000 ppm $\mathrm{CH}_{4}$.

$\mathrm{Zu}$ Beginn erkennt man einen linearen Anstieg des Messsignals, welcher nach etwa 1000 s abflacht. Der Verlauf lässt sich durch die Erwärmung der Messzelle erklären. Dem System wurde stetig Energie zugeführt, sodass es sich nicht im thermischen Gleichgewicht befindet. Über ein entsprechendes Kühlsystem könnte eine Temperaturstabilisierung erfolgen, dies wurde jedoch nicht weiter untersucht, da der Anstieg des Messsignals unabhängig von der $\mathrm{CH}_{4}$-Konzentration war und nur von der Temperaturzunahme der Messzelle abhing. Der Störeinfluss konnte deshalb über eine rechnerische Temperaturkompensation berücksichtigt werden. Es erfolgte eine Mittelung über 150 Messwerte je Messung (siehe Abb. 5).

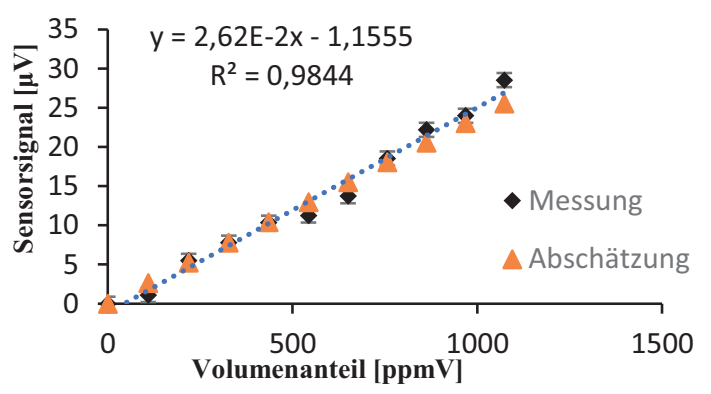

Abb. 5: Photoakustisches Signal (offsetkorrigiert) für 0-1000 ppmV $\mathrm{CH}_{4}$ in $\mathrm{N}_{2}$ bei Verwendung des offenen Strahlers.

In Abb. 5 wird die Mikrofonspannung für Volumenanteile von 0 bis $1073 \mathrm{ppmV} \mathrm{CH}_{4}$, in $\mathrm{N}_{2}$ verdünnt, dargestellt (offener Strahler). Der nach Gl. 3 zu erwartende lineare Anstieg des photoakustischen Signals mit steigendem Volumenanteil ist zu erkennen. Ebenso stimmt die Abschätzung in guter Näherung mit den experimentellen Messwerten überein. Das

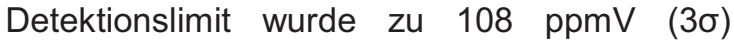
bestimmt.

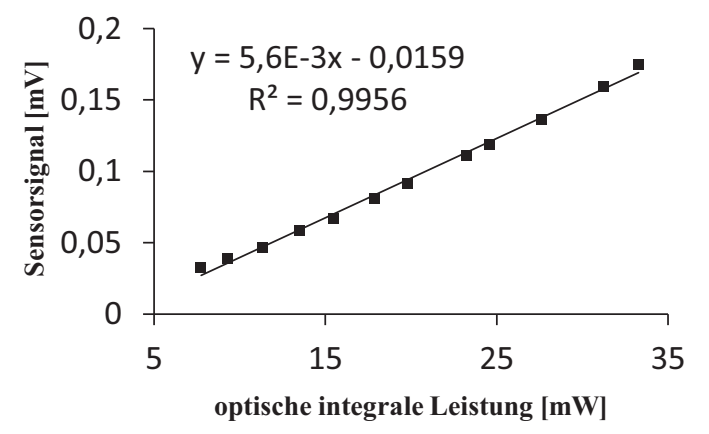

Abb. 6: Korrelation von Mikrofonspannung und optischer Leistung des offenen Strahlers für $4000 \mathrm{ppmV} \mathrm{CH}_{4}$.

Abb. 6 stellt den Zusammenhang von optischer Leistung der Strahlungsquelle und dem Anstieg des photoakustischen Signals dar. Dazu wurde 4000 ppmV $\mathrm{CH}_{4}$ in die Messkammer eingeleitet und das Signal bei 0,4 bis $1 \mathrm{~W}$ elektrischer Leistung aufgenommen. Die optische Leistung 
am Ende der Zelle wurde über ein breitbandiges optisches Leistungsmessgerät vom Typ PM16401 (Fa. Thorlabs) detektiert. Der nach GI. 3 zu erwartende lineare Zusammenhang von der optischen Strahlerleistung und dem photoakustischem Signal konnte so experimentell bestätigt werden werden.

Als zweite Strahlungsquelle wurde ein, mit $\mathrm{Kr}$ geschlossener, MEMS-Membranstrahler verwendet. Auf dem Reflektor des Strahlers wurde ein Saphirfilterglas aufgebracht, welches den Strahler hermetisch verschließt. Der Strahler konnte so direkt in die Messzelle d.h. ohne Eintrittsfenster eingesetzt werden. Auch hier wurde eine Kalibriergerade von 0 bis 1073 ppmV $\mathrm{CH}_{4}$ aufgenommen. Das Ergebnis ist in Abb. 7 dargestellt.

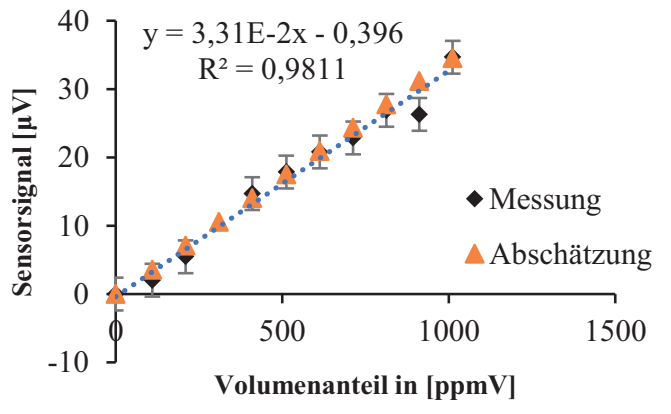

Abb. 7: Photoakustisches Signal (offsetkorrigiert) für 0-1000 ppmV $\mathrm{CH}_{4}$ in $\mathrm{N}_{2}$ bei Verwendung des geschlossenen mit Kr gefüllten Strahlers.

Die erhöhte optische Leistung spiegelt sich auch in einem größeren Anstieg der Kalibriergerade wieder. Da der Strahler nicht mehr durch ein zusätzliches Eintrittsfenster vom Gasvolumen entkoppelt ist, steigt auch das Hintergrundgeräusch, hervorgerufen durch den Strahler selbst, an. Aufgrund des hohen akustischen Grundpegels sind Messungen

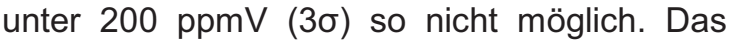
Auflösungsvermögen beträgt deshalb nur noch

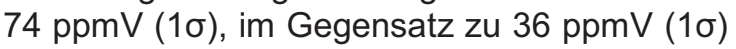
bei Einsatz des offenen Strahlers mit Eintrittsfenster.

\section{Zusammenfassung}

Es wurde ein photoakustisches Gasmesssystem bestehend aus einem elektrisch moduliertem MEMSBreitbandstrahler und einem MEMS-Mikrofon untersucht. Messungen haben gezeigt, dass die Detektion von Methan bis 108 ppmV möglich ist. In Kombination mit dem bereits bestehenden NDIR-Gasmesssystem ergeben sich so Möglichkeiten im Bereich SensorFusion, um weitere Informationen über die Gasphase zu erhalten. Da beide Verfahren im selben Sensor gleichzeitig eingesetzt werden können, kann so ein redundantes System entwickelt werden. Dies bietet die Möglichkeit, die Ausfallsicherheit des Gassensors zu erhöhen, bzw. Fehlfunktionen des Sensors zu detektieren. Dies ist insbesondere für Anwendungen zur funktionalen Sicherheit (SIL) ein denkbarer Ansatz. Aufgrund der niedrigen Modulationsfrequenz wirken sich Störgeräusche aus der Umgebung auf die Messung aus. Der Sensor sollte daher zukünftig von seiner Umgebung möglichst akustisch entkoppelt sein. Da die Zelle während der Messung verschlossen sein muss, kommt es zu einer Erwärmung des Gassensors. Idealerweise sollte dieser daher temperaturreguliert werden, oder wie in dieser Arbeit gezeigt, über eine Temperaturkompensation das Messsignal korrigiert werden. Über optische Filter kann die Selektivität für verschiedene Gase erhöht und Quereinflüsse minimiert werden.

\section{Danksagung}

Die Autoren danken dem Bundesministerium für Bildung und Forschung für die Förderung des Verbundprojektes MPAS2 (Förderkennzeichen 03ET1317B).

\section{Literaturnachweis}

[1] T. Rück, R. Bierl, Sensors and Actuators A 263 (2017), 501-509, doi:10.1016/j.sna.2017.06.036

[2] T. Tomberg, M. Vainio,T. Hieta, L. Halonen, Scientific Reports, Bd. 8, S. 1-7, doi:10.1038/s41598-018-20087-9

[3] P. Patimisco, G. Scamarcio, Frank K. Tittel, V. Spagnolo, Sensors 2014 (14), S. 6165-6206, doi: $10.3390 / \mathrm{s} 140406165$

[4] A. Bell, American Journal of Science, 118, S. 305-324, doi: 10.2475/ajs.s3-20.118.305

[5] J. Huber, A. Ambs, J. Wöllenstein, Procedia Engineering 120 (2015), S. 283-288, doi: 10.1016/j.proeng.2015.08.616

[6] J. Huber, K. Schmitt, J. Wöllenstein, J. Sens. Sens. Syst., 5 (2016), S. 293-299, doi:10.5194/jsss-5-293-2016 